\title{
Numerical Simulation of Gas Flow Based on Three-dimensional Reconstruction Using Computed Tomography
}

\author{
Xiangyu $\mathrm{Chu}^{2, \text { a }}{ }^{\text {Gang Wang }}{ }^{1,2, \mathrm{~b}}$ \\ ${ }^{1}$ Shandong University of Science and Technology, Mine Disaster Prevention and Control-Ministry of State Key \\ Laboratory Breeding Base, Qingdao 266590, PR China \\ ${ }^{2}$ Shandong University of Science and Technology, College of Mining and Safety Engineering, Qingdao \\ 266590, PR China \\ a email: 18306483235@163.com, ${ }^{\mathrm{b}}$ Corresponding author email: gang.wang@ sdust.edu.cn
}

\begin{abstract}
In order to obtain the real physical model characterizing the pore and fissure structures of coal rocks and explore the seepage rules of coalbed methane (CBM) using numerical simulation, we proposed a sequential study method from real coal model to coal CAD model to coal finite element model. The coal sample from Changzhi Shanxi was scanned using high precision $\mu \mathrm{CT} 225 \mathrm{kVFCB} C \mathrm{CT}$ system and the obtained CT data were subject to threshold segmentation using the compensated DTM method. The real model of coal is converted to the CAD model of coal by reverse engineering, Lastly, The coal body finite element model established by Ansys as an example, the seepage of coal gas were simulated, analyzed coal pore pressure and velocity distribution, the permeability coefficient of $X$, $Y$, along the $Z$ direction and calculation. Calculation results show that the micro scale $(<100 \mu \mathrm{m})$ coal permeability anisotropy, which is affected by coal body structure is obvious. The numerical simulation results show that with the increase of the pressure gradient at the micro scale, the penetration velocity of the gas in the fracture section is gradually increased.
\end{abstract}

Keywords - CT; three-dimensional reconstruction; DTM; reverse engineering; gas flow

\section{INTRODUCTION}

With the continuous increase of oil and gas demands and the ever-worsening of atmospheric pollution, coalbed methane $(\mathrm{CBM})$ as a clean energy resource has entered a new and rapid development stage. CBM is not only an accompany gas in the coal-forming process, but also one of the main factors causing coalmine accidents. CBM seepage means the flow of CBM in the coal bed. Studies on the characteristics of CBM seepage in the coal bed are of practical significance for scientific development and utilization of CBM.

Since most coals are buried deeply underground, it is greatly difficult to study coals through observation method and field survey. Researchers mainly studied microstructure of coals based on experiment. CT method can not only acquire parameters that represent pore structure of coals [1], but also give a quantitative characterization of micro-pore structures of coals while protecting coal mass as much as possible and establish a visible realistic model. Nie et al [2] discussed meso-scaled coal fracture process by using a designed CT system. Cai et al [3] explored fractures caused by coal destruction under triaxial compression and its impact on coal osmotic coefficient by combining CT reconstruction technology with acoustic emission and ultrasonic technique.
The characteristics of porous structure in coal are related to the occurrence state of the gas, also influence its and the interaction between gas and liquid molecules, for unconventional oil and gas resources development [4], CO2 geological storage and underground hydrology research [5] has far-reaching significance. In recent years, researchers have made a certain progress research of fracture seepage. Teng et al. [6] and Jin et al. [7] used of LBM method, seepage migration law of fractures in the coal gas are simulated. Gong et al. [8] based on the structural fissure and cleat discrete crack model, put forward a new method for two-dimensional gas recovery simulation. Mi et al. [9] based on multi-scale discrete fracture model obtained the micro nano scale matrix block gas diffusion mechanism for shale gas production distribution and reservoir pressure has a greater impact.

However, neither experimental researches nor numerical simulations mentioned above analyzed and discussed the gas flow in coal from the microscopic point of view. In this study, combined the numerical simulation with $3 \mathrm{D}$ CT reconstruction technique to study the CBM flow in coals. First, the real micro-scale slices of coal samples were obtained using CT and reconstructed as the real 3D model of pore and fissure structures of coal rocks. Then the obtained real 3D model was further converted first into the coal's CAD model then into the coal's finite element model, thus achieving the real-model-based finite element numerical simulation. The simulation is obviously different from the simplified ideal-model-based numerical simulation. The numerical simulation experiment of gas flow in coal was carried out, and the law of gas flow in porous structure of coal was studied.

\section{MATERIALS AND METHODS}

\subsection{CT experimental system}

The coal sample was scanned using $\mu \mathrm{CT} 225 \mathrm{kVFCB}$ high-precision CT system from Taiyuan University of Technology. Before scanning, coal samples were prepared as cylinders with 
cross-section diameter less than $3 \mathrm{~mm}$. When performing CT scanning, samples were magnified 100-fold and the minimum observable pore diameter was $1.94 \mu \mathrm{m}$. The CT scanned slice size was 2041 pixels $\times 2041$ pixels.

\subsection{Digital terrain model (DTM) threshold segmentation}

In the CT image, changes in the gray value reflects the internal density changes of the objects. White color means the highest gray value and object density and indicates the position of coal's skeleton, while black color means the lowest gray value and object density and reveals the position of pores or fractures. Gray color is in between the white and the black. Because most pixels of CT images are gray, threshold segmentation is to select an appropriate gray value and convert the CT images into binary images with only black and white colors. In detail, a CT image can be considered as a matrix composed of gray values. If the gray value of each point in the CT image is regarded as the terrain height values, each gray scale image can be considered as a digital terrain model (DTM). Because the algorithm of DTM threshold segmentation method has been described in detail previously
[10-12].

For any CT image, the relationship of porosity to its corresponding gray value can be obtained. The essence of calculating the surface porosity of the image is to obtain the minimum value of the gray value and the porosity function [10-12]. In practice, sometimes the ideal porosity curve is unable to find because either the curve has no minimum value or the porosity corresponding to the minimum value is inconsistent with the actual porosity and has greater deviation. This phenomenon is main caused by the noise in $\mathrm{CT}$ image.

The noise in CT image is an objective existence. It is mainly results from the deviation of obtained images from actual images due to CT machine system itself and its environment, thus de-noising CT images can improve their accuracy. Noise identification of CT image found that most noises in CT images are Gaussian noise and could be well removed using the Wiener filter.

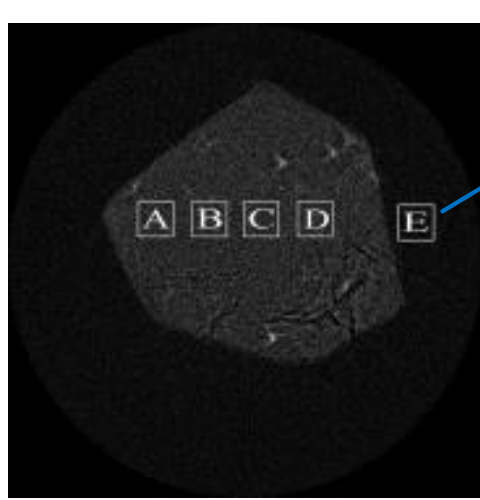

(a) A CT slice of coal spicemen

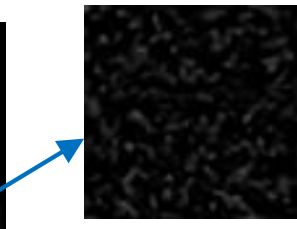

(b) before denoise

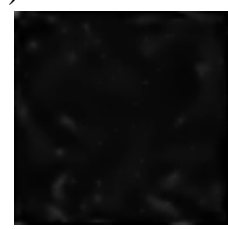

(c) after denoise

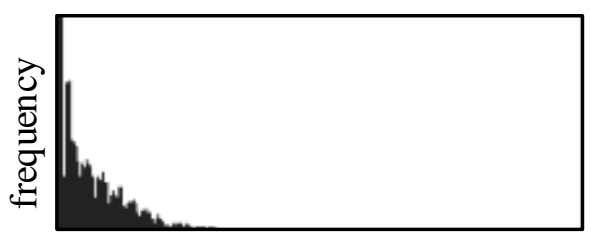

(d) gray histogram before denoise gray level

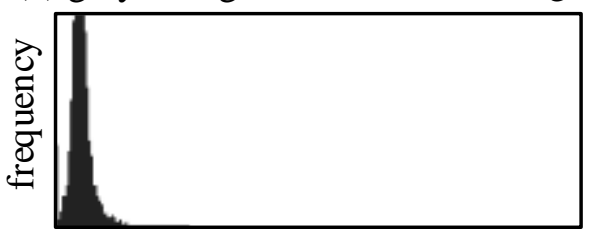

(e) gray histogram before denoise gray level

Fig. 1 CT slices of coal specimen and comparison before and after denoising

After de-noising, the thresholds of CT images of the coal sample can be obtained using the DTM threshold segmentation method. Fig. 1 shows the DTM threshold segmentation of the CT images of blind coal from Changzhi Shanxi. To further reduce the calculation pressure due to a large amount of data, region A with 100 pixels $\times 100$ pixels of the image was denoised and DTM threshold segmentation was conducted for the CT images of the 100 slices in the middle of the coal column.

The actual porosity curve obtained using DTM threshold segmentation contains multiple minimal points, respectively corresponding to multiple different porosities. In order to obtain the minimal point representing the real porosity, it is necessary to verify the correctness of the DTM threshold segmentation method using 3D reconstruction method.

To reduce the regional structure contingency of CT image and make the data more representative, $100 \mathrm{CT}$ slices of each region $\mathrm{A}, \mathrm{B}, \mathrm{C}$ and $\mathrm{D}$ in Fig. 1(a) were subjected to statistical analysis. Then the minimum $\varepsilon_{\text {min }}$, average $\varepsilon_{\text {ave }}$, and maximum $\varepsilon_{\max }$ of the minimal values of the porosity curve of all CT slices were obtained and their corresponding min, ave, and max horizontal coordinate values were obtained. According to the thresholds corresponding to the overall averages, the $3 \mathrm{D}$ reconstructions were carried out based on the slices of each of the four regions, respectively, and the porosities of reconstructed models were statistically analyzed and shown in Table 1.

From Table 1 it can be seen that with the minimum value increasing, the porosity obtained with the DTM threshold segmentation gradually approaches the porosity obtained using the 3D reconstruction. When the minimum takes its maximum, the porosities obtained through both methods differ by less than $4 \%$. In other words, the maximum among minimum of one CT slice can be considered as the real porosity of coal reflected by the CT image, and its corresponding abscissa is the threshold obtained with the DTM threshold segmentation. 
Table 1 DTM threshold segmentation results and porosity of three-dimensional reconstruction model

\begin{tabular}{ccccc}
\hline \multirow{2}{*}{$\begin{array}{c}\text { Statistical } \\
\text { area }\end{array}$} & \multicolumn{2}{c}{ DTM threshold segmentation method } & \multicolumn{2}{c}{$\begin{array}{c}\text { Three-dimensional } \\
\text { reconstruction }\end{array}$} \\
\cline { 2 - 5 } A area & Value type of minimum & Threshold & porosity /\% & porosity /\% \\
& minimum & 18 & 11.23 & 0.19 \\
& average & 32 & 15.97 & 3.89 \\
maximum & minimum & 46 & 19.36 & 21.55 \\
\hline \multirow{3}{*}{ area } & average & 19 & 9.77 & 0.12 \\
& maximum & 38 & 16.07 & 4.04 \\
C area & minimum & 50 & 19.15 & 20.90 \\
& average & 15 & 9.69 & 0.09 \\
Darea & maximum & 28 & 15.63 & 2.89 \\
& minimum & 43 & 18.97 & 19.50 \\
\hline
\end{tabular}

\subsection{Coal sample reconstruction}

The porosity of coal is about 3\% 25\% [13,14]. Correct understanding on porous structural features of coal mass is the basis to study fracture seepage. Porous structure of coal mass is very small and is difficult to be observed by conventional test method. Distribution of porous structure in two-dimensional plane could be acquired through CT-based tomographic scanning of the coal mass. The three-dimensional porous structure could be gained from volume data formed through spatial pileup of a series of two-dimensional sections and data interpolation. This is known as three-dimensional reconstruction. If two-dimensional sections used CT sections, the whole process can be called as the CT three-dimensional reconstruction [15] This process is shown in Fig. 2.

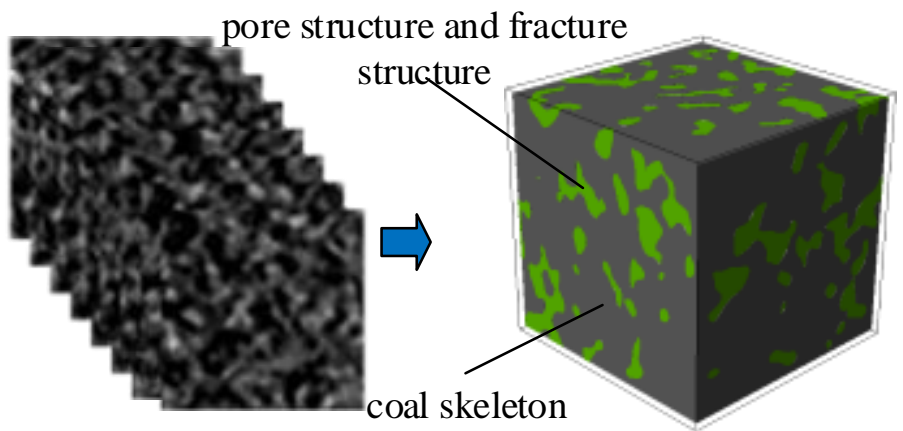

Fig. 2 Process of 3D reconstruction b ased on computed tomography

Four areas A, B, C and D as shown in Fig.2(a) of 100 pixels $\times 100$ pixels were selected for $3 \mathrm{D}$ reconstruction of pores and fractures in coals. The obtained 3D model was 100 pixels $\times 100$ pixels $\times 100$ pixels while the actual samples were $0.19 \mathrm{~mm} \times 0.19 \mathrm{~mm} \times 0.19 \mathrm{~mm}$.

\subsection{Reverse engineering technique and CAD digital model}

To make the 3D reconstructed model able to introduce the mainstream software for more in-depth research, it is necessary to optimize the model, vectorize the surface model and create the CAD digital model. Today's mainstream software, such as Ansys, COMSOL, Abaqus, and the like, all support the CAD digital model based on the NURBS surface data [16] and the reverse engineering technique provides a method to convert a real model (CT 3D data) into a CAD model.
The Geomagic reverse engineering technique software was applied to convert the real model of the coal into the CAD digital model. The conversion process is as follows: 1) read the $3 \mathrm{D}$ reconstructed STL file, as shown in Fig. 3(a); 2) repair possible existent topology errors and make the surface smooth, as shown in Fig. 3 (b) [17]; 3) extract the contours of the 3D model, as shown in Fig. 3 (c); 4) use the contours as the boundaries of the model and divide the entire 3D model into multiple surface patches, as shown in Fig. 3 (d); 5) further divide these surface patches to form a grid, as shown in Fig. $3(\mathrm{e})$; 6) fit to generate NURBS surfaces, as shown in Fig. 3 (f), and 7) form the CAD digital model and complete the conversion of a real model into the CAD digital model. 


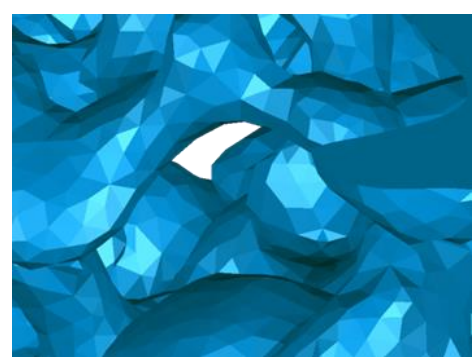

(a) The initial state

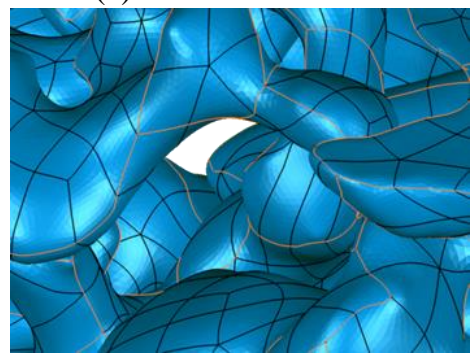

(d) Construct patches

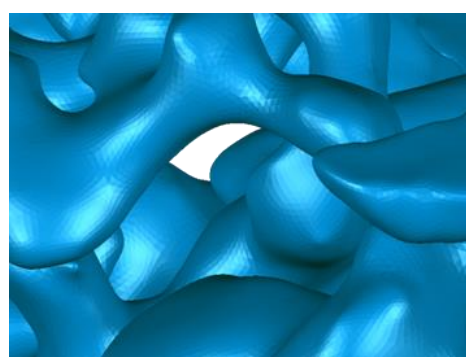

(b) Surface repair

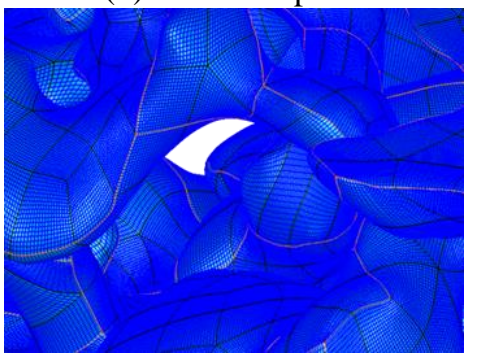

(e) Construct grids

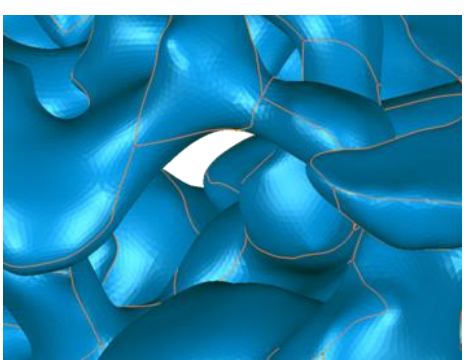

(c) Detect Contours

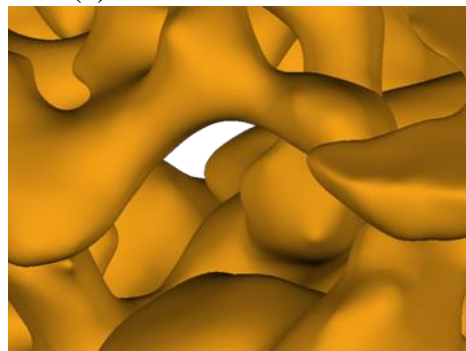

(f) Fit surfaces.

Fig. 3 The reverse engineering optimization

\subsection{The establishment of finite element model}

The governing equation should apply the most basic governing equation because this numerical simulation focuses on the gas seepage in coal fractures. Ignoring the density fluctuation, we can consider gas seepage as an unsteady incompressible viscous flow that follows the laws of mass conservation, momentum conservation, and energy conservation. The fluid flow of single fracture is often described by the well-known Navier-Stokes (N-S) equation [18] as follows:

$\rho\left[\frac{\partial \boldsymbol{v}}{\partial t}+(\boldsymbol{v} \cdot \nabla) \boldsymbol{v}\right]=\rho \boldsymbol{f}-\nabla p+\mu \nabla^{2} \boldsymbol{v}$ $\nabla p$ is the pressure gradient force and $\mu \nabla^{2} v$ is the viscous force.

Boundary conditions refer to the dependent variables on the boundaries of the solution domain or the variation law of the first-order derivative of the dependent variables with positions and time. All CFD problems require boundary conditions and transient problems need initial conditions. The flow field has different solving methods and treatments of boundary and initial conditions. The geometric model was preprocessed by the CFX software. The boundary conditions of the realistic models of coal mass are presented in Fig. 4.

where the left side is the inertia force, $\rho f$ is the body force,

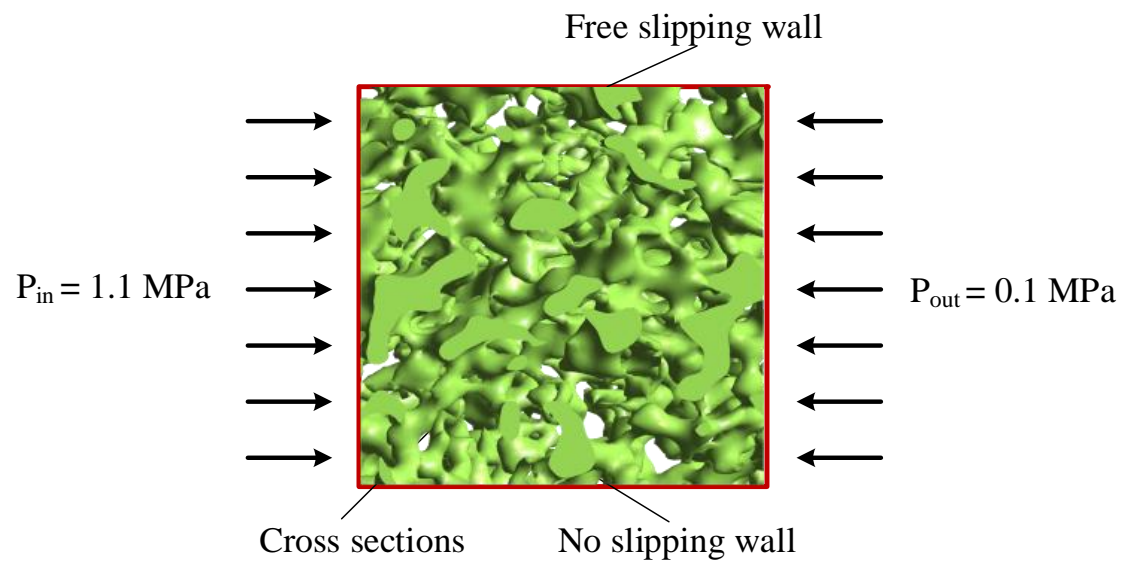

Fig.4 Boundary conditions settings 

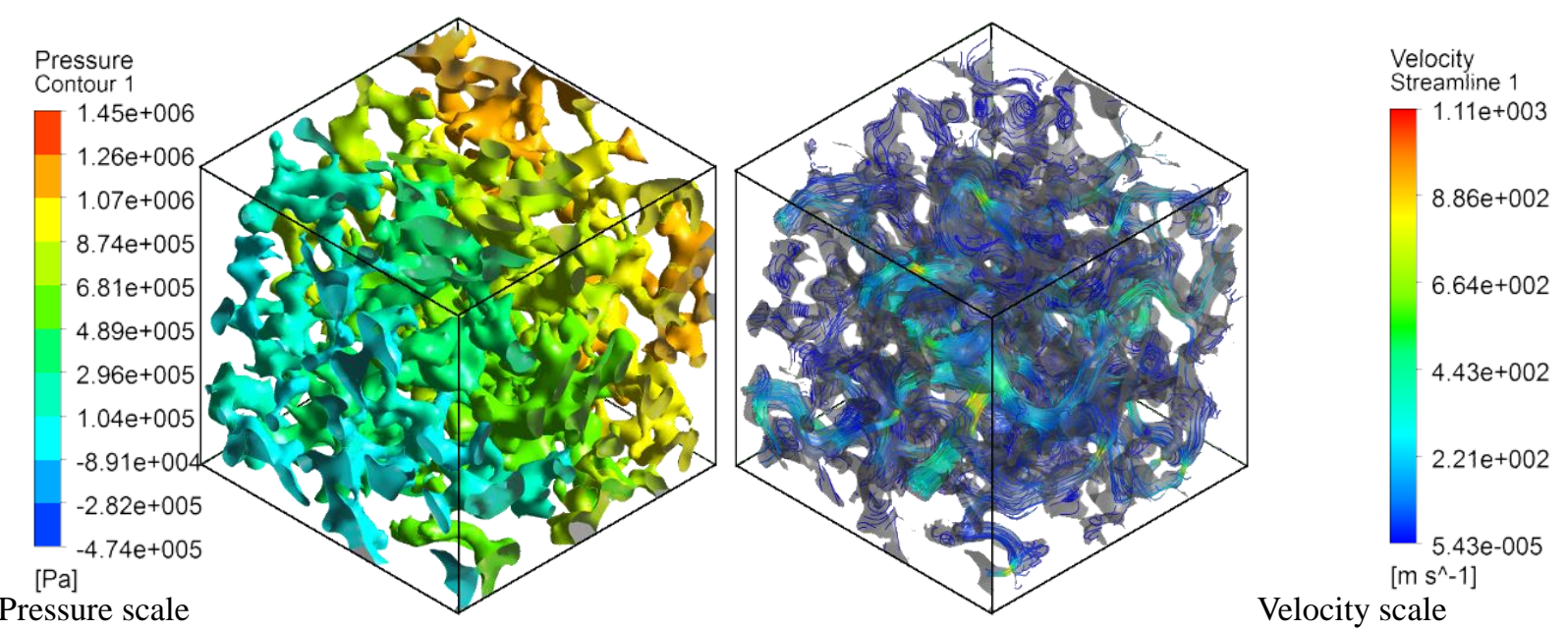

Fig. 5 Pressure contour of coal pore and gas streamline

III.RESULTS AND DISCUSSION

The established models were quantized and solved ,the A, $\mathrm{B}, \mathrm{C}, \mathrm{D}$ four areas in the $\mathrm{X}, \mathrm{Y}, \mathrm{Z}$ three directions of coal pore pressure distribution and flow field velocity distribution are obtained as shown in Fig. 5.

When the simulation results post processing, in order to facilitate comparison, the unified pressure scale and speed scale are set up. The permeability coefficient was calculated by the formula (2) Darcy equation. The inlet boundary mass flow can be obtained directly from the post processing data, and the volume flow can be obtained by the combination of the methane density. The formula (1) is brought into the Darcy equation and the formula (3) is obtained. $G$ is the mass flow, $\mathrm{kg} \bullet \mathrm{s}^{-1} ; A$ is the flow area, $\mathrm{m}^{2} ; g$ is the acceleration of gravity, $\mathrm{m} \bullet \mathrm{s}^{-2} ; \nabla l$ is the distance, $\mathrm{m} ; \nabla p$ is the pressure difference on both ends, $\mathrm{Pa}$.

$$
Q=\frac{G}{\rho}
$$

(2)

$$
\begin{aligned}
& \frac{Q}{A}=K \frac{\Delta h}{\Delta l} \\
& K=\frac{G}{A} \cdot \frac{g \Delta l}{\Delta P}
\end{aligned}
$$

According to the established finite element model, the values of $g, \nabla l$ and $\nabla p$ are constant in each calculation of the coefficient of permeability. The permeability coefficient is determined by the value of $G$ and $A$, and the values of the permeability coefficient and other parameters are calculated as shown in table 2.

Table 2 Hydraulic conductivity of coal in different seepage directions

\begin{tabular}{ccccc}
\hline $\begin{array}{c}\text { Reconstruction } \\
\text { area }\end{array}$ & Seepage direction & $\begin{array}{c}\text { The mass flow of } \\
\text { inlet } / \mathrm{kg} \cdot \mathrm{s}^{-1}\end{array}$ & Outlet area $/ \mathrm{m}^{2}$ & $\begin{array}{c}\text { Permeability } \\
\text { coefficient } / \mathrm{m} \cdot \mathrm{s}^{-1}\end{array}$ \\
\hline & $\mathrm{X}$ & $8.56 \mathrm{E}-08$ & $2.97 \mathrm{E}-09$ & $3.35 \mathrm{E}-08$ \\
$\mathrm{Y}$ & $5.96 \mathrm{E}-08$ & $2.76 \mathrm{E}-09$ & $2.19 \mathrm{E}-08$ \\
& $\mathrm{Z}$ & $6.12 \mathrm{E}-08$ & $2.52 \mathrm{E}-09$ & $2.92 \mathrm{E}-08$ \\
& $\mathrm{X}$ & $1.21 \mathrm{E}-07$ & $2.49 \mathrm{E}-09$ & $4.22 \mathrm{E}-08$ \\
B & $\mathrm{Y}$ & $7.21 \mathrm{E}-08$ & $3.33 \mathrm{E}-09$ & $2.52 \mathrm{E}-08$ \\
& $\mathrm{Z}$ & $5.38 \mathrm{E}-08$ & $1.58 \mathrm{E}-09$ & $3.62 \mathrm{E}-08$ \\
& $\mathrm{X}$ & $9.52 \mathrm{E}-08$ & $2.36 \mathrm{E}-09$ & $4.25 \mathrm{E}-08$ \\
$\mathrm{Y}$ & $\mathrm{Y}$ & $1.24 \mathrm{E}-07$ & $2.21 \mathrm{E}-09$ & $6.31 \mathrm{E}-08$ \\
& $\mathrm{Z}$ & $1.13 \mathrm{E}-07$ & $1.61 \mathrm{E}-09$ & $8.25 \mathrm{E}-08$ \\
& $\mathrm{X}$ & $7.23 \mathrm{E}-08$ & $1.53 \mathrm{E}-09$ & $4.68 \mathrm{E}-08$ \\
& $\mathrm{Y}$ & $3.81 \mathrm{E}-08$ & $2.27 \mathrm{E}-09$ & $1.29 \mathrm{E}-08$ \\
\end{tabular}

According to the simulation results, along the direction of gas flow, the pressure of coal pore is gradually reduced, and the gas flow velocity gradually becomes larger. When the pore channel suddenly shrinks, the gas flow rate increases suddenly, the pore pressure decreases and then increases. The pressure change is small, and the color change is not obvious in the change of pore channel flat region.

Permeability coefficient as a macroscopic parameter of coal seepage, in microscopic scale the difference of structure can make the difference of permeability coefficient larger, but the difference is still in the same order of magnitude. The difference of permeability coefficient of coal body in different seepage directions in the four regions shows that the permeability coefficient of coal is anisotropic in the micro scale.

After three dimensional reconstruction of the CT region of $\mathrm{B}$, the model of the coal body is obtained, and the obvious fracture structure in the coal body are found as shown in Figure 6. 


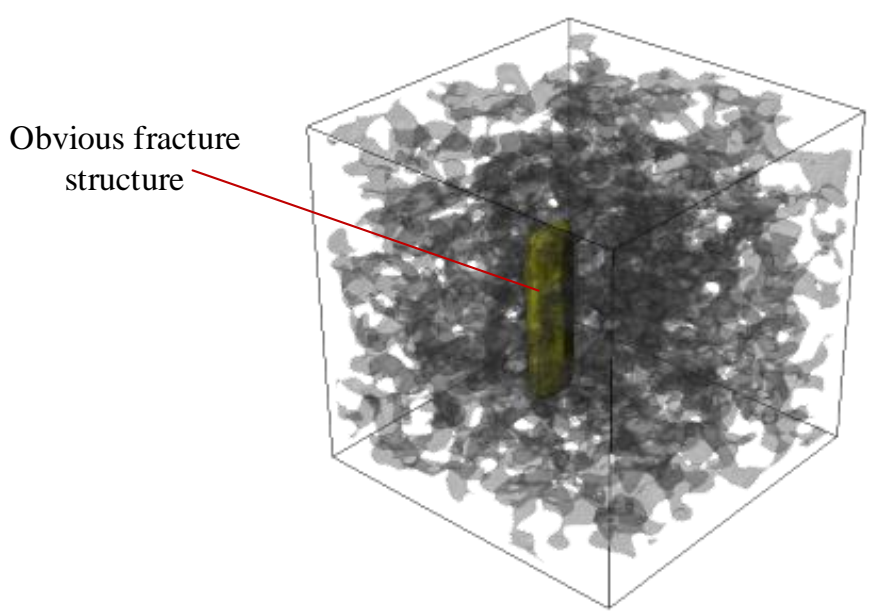

Fig. 6 Real coal model of B

Table 3 The inlet pressure and penetration velocity at specific pressure gradient

\begin{tabular}{ccccccccc}
\hline $\begin{array}{c}\text { Inlet } \\
\text { pressure } \\
(\mathrm{MPa})\end{array}$ & $\begin{array}{c}\text { Pressure difference } \\
\text { between inlet and } \\
\text { outlet }(\mathrm{MPa})\end{array}$ & $\begin{array}{c}\text { Pressure } \\
\text { gradient } \\
\left(\mathrm{E}^{9} \mathrm{MPa} \cdot \mathrm{m}^{-1}\right)\end{array}$ & 1 & 3 & 5 & 7 & $\begin{array}{c}\text { penetration velocity of section }\left(\mathrm{m} \cdot \mathrm{s}^{-1}\right) \\
9\end{array}$ & $\begin{array}{c}\text { Average } \\
\text { penetration } \\
\text { velocity }\left(\mathrm{m}^{-1} \mathrm{~s}^{-1}\right)\end{array}$ \\
\hline 0.3 & 0.2 & 0.8 & 115.2 & 128.3 & 129.1 & 126.2 & 126.9 & 125.14 \\
0.7 & 0.6 & 2.5 & 203.2 & 213.5 & 219.8 & 217.2 & 213.2 & 213.38 \\
1.1 & 1 & 4.2 & 292.1 & 300.5 & 288.3 & 279.4 & 293.2 & 290.7 \\
1.5 & 1.4 & 5.9 & 331.5 & 343.7 & 342.2 & 348.1 & 352.1 & 343.52 \\
1.9 & 1.8 & 7.6 & 385.6 & 410.1 & 433.1 & 399.3 & 398.2 & 405.26 \\
2.3 & 2.2 & 9.2 & 435.2 & 459.1 & 466.5 & 454.9 & 437.6 & 450.66 \\
2.7 & 2.6 & 10.9 & 495.2 & 523.2 & 524.2 & 510.1 & 492.5 & 509.04 \\
\hline
\end{tabular}

In order to further study the flow law of gas in the fracture, in the post processing, the fracture part in the model between the flow inlet and outlet is selected as the research object. Under the different pressure gradient, the penetration velocity of 5 sections of the fracture section was extracted. Inlet boundary pressure and the penetration velocity of the cross section under the corresponding pressure gradient is shown in table 2.It can be seen from the Table 2, with the increase of the pressure gradient, the gas penetration velocity of section is gradually increased.

\section{IV.CONCLUSIONS}

1) The real-model-based finite element numerical simulation is achieved by converting the coal real model first into the CAD model then into the finite element model on the basis of CT-based $3 \mathrm{D}$ reconstruction and reverse engineering techniques.

2) The DTM threshold segmentation method calculates the threshold and porosity using mathematical statistics method from the objective point of view. The method is universal and unaffected by instrument and environment Its threshold is the abscissa $\mathrm{X}$ value corresponding to the maximum of minimum on the porosity curve. The verification of the method's correctness through the $3 \mathrm{D}$ reconstruction method indicates the obtained porosities differ by less than $4 \%$ and is acceptable.
3) Computational fluid dynamics in the introduction of CT three-dimensional reconstruction technology is becoming more and more mature, making in solving multi-scale flow coruscate gives new vitality, especially in the world is facing the situation of energy restructuring, for scientific guidance for gas and other unconventional oil and gas resources development has important significance.

4) The real-model-based finite element numerical simulation can simplify the micro pore structure of coal, describe the spatial distribution of gas seepage velocity field and pressure field directly, and can be used to calculate the permeability coefficient of coal body. The permeability coefficient is determined by the property of the coal pore medium and the fluid, and the permeability coefficient of the coal body needs further experimental verification.

\section{ACKNOWLEDGEMENT}

The authors would like to acknowledge the support of National Natural Science Foundation of China (Project No. 51304128, 51304237), the support of National Natural Science Foundation of Shandong Province (Project No. ZR2013EEQ015) and the Program for the Outstanding Young 
Scientists of Shandong University of Science and Technology (Project No. 2015JQJH105).

\section{REFERENCES}

[1] Yao Y, Liu D, Che Y, et al. Non-destructive characterization of coal samples from China using micro focus X-ray computed tomography [J]. International Journal of Coal Geology, 2009, 80(2): 113-123.

[2] Nie B, He X, Li X, et al. Meso-structures evolution rules of coal fracture with the computerized tomography scanning method $[\mathrm{J}]$. Engineering Failure Analysis, 2014, 41: 81-88.

[3] Cai Y D, Liu D M, Mathews J P, et al. Permeability evolution in fractured coal Combining triaxial confinement with X-ray computed tomography, acoustic emission and ultrasonic techniques [J]. International Journal of Coal Geology, 2014, 122: 91-104.

[4] Li T, Wu C, Liu Q. Characteristics of coal fractures and the influence of coal facies on coalbed methane productivity in the South Yanchuan Block, China [J]. Journal of Natural Gas Science and Engineering, 2015, 22: 625-632.

[5 Rong H, Bai H. Pore structure characteristics of the relative water-resisting layer on the top of the Ordovician in Longgu Coal Mine [J]. International Journal of Mining Science and Technology, 2014, 24(5): 657-661.

[6].Teng G R, Tan Y L, Gao M. Simulation of gas seepage in fissured coal based on lattice Boltzmann method [J]. Chinese Journal of Rock Mechanics and Engineering, 2007, 36(Supp.1): 3503-3508.

[7] Jin Y, Zhu Y B, Wu Y, et al. Numerical investigation of migration mechanism for coal-bed methane flow through cleats with rough surfaces in coal reservoir [J]. Journal of China coal society, 2014, 39(9): 1826-1834.

[8] Gong B, Zhang Y, Fan Y, et al. A novel approach to model enhanced coal bed methane recovery with discrete fracture characterizations in a geochemical simulator [J]. Journal of Petroleum Science and Engineering,
2014, 124: 198-208.

[9] Mi L, Jiang H, Li J. The impact of diffusion type on multiscale discrete fracture model numerical simulation for shale gas $[\mathrm{J}]$. Journal of Natural Gas Science and Engineering, 2014, 20: 74-81.

[10] Taud H, Martinez-Angeles R, Parrot J F, et al. Porosity estimation method by X-ray computed tomography [J]. Journal of Petroleum Science and Engineering, 2005, 47(3-4): 209-217.

[11] Yuan Z X, Mao L T, Zhao D. Computation method on 3D porosity of soil microstructure based on digital terrain model[J]. Journal of Liaoning Technical University (Natural Science), 2011, 30(5): 734-737.

[12] Mao L T, Shi P, Tu H , et al. Coal damage calculation under uniaxial loading based on CT images[J]. Journal of Liaoning Technical University (Natural Science), 2013, 32(1): 59-63.

[13] Wang J F. Percolation percolation characteristics of heterogeneous porous media [D]. Taiyuan University of Technology, Taiyuan, 2011.

[14] Ma P L. Manual of coal mine gas disaster prevention and control technology [M]. Chemical Industry Press, Beijing, 2007.

[15] Bodla K K, Garimella S V, Murthy J Y. 3D reconstruction and design of porous media from thin sections [J]. International Journal of Heat and Mass Transfer, 2014, 73: 250-264.

[16] YOO D J. Three-dimensional surface reconstruction of human bone using a B-spline based interpolation approach [J]. Computer-Aided Design, 2011, 43: 934-947.

[17] LI C S, Zhang D, Wang H X, et al. 3D mesh generation for soil-rock mixture based on $\mathrm{CT}$ scanning $[\mathrm{J}]$. Rock and Soil Mechanics, 2014, 35(9): 2731-2736.

[18] Ding Z. R., Fluid mechanics [M]. Higher Education Press, Beijing, 2003. 